\title{
Brain network connectivity differs in early-onset neurodegenerative dementia OPEN
}

Massimo Filippi,

MD, FEAN

Silvia Basaia, MSc

Elisa Canu, PhD

Francesca Imperiale, $\mathrm{PhD}$

Alessandro Meani, MSc

Francesca Caso, MD,

$\mathrm{PhD}$

Giuseppe Magnani, MD

Monica Falautano, MSc

Giancarlo Comi, MD

Andrea Falini, MD

Federica Agosta, MD, $\mathrm{PhD}$

Correspondence to

Prof. Filippi:

filippi.massimo@hsr.it

Editorial, page 1758

Supplemental data at Neurology.org

\section{ABSTRACT}

Objective: To investigate functional brain network architecture in early-onset Alzheimer disease (EOAD) and behavioral variant frontotemporal dementia (bvFTD).

Methods: Thirty-eight patients with bvFTD, 37 patients with EOAD, and 32 age-matched healthy controls underwent 3D T1-weighted and resting-state fMRI. Graph analysis and connectomics assessed global and local functional topologic network properties, regional functional connectivity, and intrahemispheric and interhemispheric between-lobe connectivity.

Results: Despite similarly extensive cognitive impairment relative to controls, patients with EOAD showed severe global functional network alterations (lower mean nodal strength, local efficiency, clustering coefficient, and longer path length), while patients with bvFTD showed relatively preserved global functional brain architecture. Patients with bvFTD demonstrated reduced nodal strength in the frontoinsular lobe and a relatively focal altered functional connectivity of frontoinsular and temporal regions. Functional connectivity breakdown in the posterior brain nodes, particularly in the parietal lobe, differentiated patients with EOAD from those with bvFTD. While EOAD was associated with widespread loss of both intrahemispheric and interhemispheric functional correlations, bvFTD showed a preferential disruption of the intrahemispheric connectivity.

Conclusions: Disease-specific patterns of functional network topology and connectivity alterations were observed in patients with EOAD and bvFTD. Graph analysis and connectomics may aid clinical diagnosis and help elucidate pathophysiologic differences between neurodegenerative dementias. Neurology ${ }^{\circledR}$ 2017;89:1764-1772

\section{GLOSSARY}

AD = Alzheimer disease; bvFTD = behavioral variant of frontotemporal dementia; CDR-SB $=$ Clinical Dementia Rating Scale-Sum of Boxes; DMN = default mode network; EOAD = early-onset Alzheimer disease; FTLD = frontotemporal lobar degeneration; LCW = lobar connectivity weight; MMSE = Mini-Mental State Examination; NBS = network-based statistics; RS = resting-state; $\mathbf{S N}$ = salience network.

The frequent clinical overlap in early-onset Alzheimer disease (EOAD) and the behavioral variant of frontotemporal dementia (bvFTD) poses serious problems in differential diagnosis with the consequence that they can be misdiagnosed, even in the most expert centers. ${ }^{1}$

Brain network connectivity is sensitive to neurodegeneration and may be a useful noninvasive dementia biomarker. Decreased functional connectivity within the default mode network $(\mathrm{DMN})$, which is activated during internal tasks such as daydreaming, envisioning the future, and retrieving episodic memories, has been associated with late-onset Alzheimer disease $(\mathrm{AD})^{2,3}$ as well as with EOAD in a few studies. ${ }^{4,5}$ In bvFTD, multiple studies have described reduced connectivity in the salience network $(\mathrm{SN})$, which is critical for social-emotionalautonomic processing, when compared with controls or patients with $\mathrm{AD} .^{6-8}$

\footnotetext{
From the Neuroimaging Research Unit (M. Filippi, S.B., E.C., F.I., A.M., F.C., F.A.), Department of Neurology (M. Filippi, G.M., M. Falautano, G.C.), Institute of Experimental Neurology, Division of Neuroscience, and Department of Neuroradiology and CERMAC (A.F.), Division of Neuroscience, San Raffaele Scientific Institute, Vita-Salute San Raffaele University, Milan, Italy.

Go to Neurology.org for full disclosures. Funding information and disclosures deemed relevant by the authors, if any, are provided at the end of the article. The Article Processing Charge was funded by San Raffaele Scientific Institute, Milan, Italy.

This is an open access article distributed under the terms of the Creative Commons Attribution-NonCommercial-NoDerivatives License 4.0 (CC BY-NC-ND), which permits downloading and sharing the work provided it is properly cited. The work cannot be changed in any way or used commercially without permission from the journal.
} 
Nevertheless, functional connectivity approaches used so far do not provide information about how networks are embedded and interact in the complex brain system. With the advent of graph network analysis, it is now possible to model and characterize topologically, from a local to a global level, the functional integrative bases that support brain behaviors. ${ }^{9}$ In late-onset $\mathrm{AD}$, graph network studies demonstrated a widespread alteration of brain topologic properties. ${ }^{10}$ In 2 studies of bvFTD, graph network abnormalities were found in frontoinsular, temporal, and subcortical regions. ${ }^{11,12}$ It remains to be determined whether this emerging technique can provide neural substrates explaining the diversity between bvFTD and EOAD. We predicted a marked pattern of network alterations including both interhemispheric and intrahemispheric connections within and outside the DMN in patients with EOAD, reflecting their multidomain cognitive and behavioral impairments. On the other hand, bvFTD would be characterized by a focal disconnectivity involving intrahemispheric, frontotemporal connections.

Table 1 Demographic and clinical findings in healthy controls (HC), patients with behavioral variant of frontotemporal dementia (bvFTD), and patients with early-onset Alzheimer disease (EOAD)

\begin{tabular}{|c|c|c|c|c|c|c|}
\hline & $\mathrm{HC}$ & EOAD & bvFTD & $\begin{array}{l}\text { p: EOAD } \\
\text { vs HC }\end{array}$ & $\begin{array}{l}\text { p: bvFTD } \\
\text { vs HC }\end{array}$ & $\begin{array}{l}\text { p: EOAD } \\
\text { vs bvFTD }\end{array}$ \\
\hline $\mathbf{N}$ & 32 & 37 & 38 & - & - & - \\
\hline Age at MRI, y & $62.3 \pm 2.6(58.9-67.0)$ & $62.1 \pm 3.9(55.2-69.1)$ & $63.8 \pm 7.3(45.5-77.4)$ & 1.00 & 0.70 & 0.46 \\
\hline Sex, F/M & $17 / 15$ & $14 / 24$ & $23 / 14$ & 0.45 & 0.17 & 0.03 \\
\hline Education, y & $14.3 \pm 4.7(5-24)$ & $10.5 \pm 4.6(3-18)$ & $9.6 \pm 3.6(4-17)$ & 0.001 & $<0.001$ & 1.00 \\
\hline Disease duration, y & - & $3.5 \pm 2.0(0.6-7.9)$ & $3.1 \pm 2.0(0.6-8.1)$ & - & - & 0.40 \\
\hline CDR & - & $1.0 \pm 0.5(0.5-3)$ & $1.0 \pm 0.57(0.5-2)$ & - & - & 0.75 \\
\hline CDR-SB & - & $4.4 \pm 1.4(2.5-8.5)$ & $4.8 \pm 2.5(1-9.5)$ & - & - & 0.60 \\
\hline NPI & - & $16.3 \pm 14.3(3-57)$ & $27.0 \pm 15.8(11-60)$ & - & - & 0.05 \\
\hline \multicolumn{7}{|l|}{ Global cognition } \\
\hline MMSE & $29.3 \pm 0.8(28-30)$ & $19.3 \pm 4.9(7-27)$ & $22.7 \pm 5.8(8-29)$ & $<0.001$ & $<0.001$ & 0.01 \\
\hline \multicolumn{7}{|l|}{ Verbal and spatial memory } \\
\hline Rey list: immediate recall & $45.1 \pm 9.0(26-58)$ & $21.0 \pm 12.0(3-40)$ & $21.0 \pm 10.4(6-41)$ & $<0.001$ & $<0.001$ & 1.00 \\
\hline Rey List: delayed recall & $8.7 \pm 3.5(3-14)$ & $1.3 \pm 1.4(0-4)$ & $3.3 \pm 3.2(0-10)$ & $<0.001$ & $<0.001$ & 0.25 \\
\hline Rey list: recognition & $14.5 \pm 0.9(12-15)$ & $5.5 \pm 4.8(2-12)$ & $12.4 \pm 2.8(7-15)$ & $<0.001$ & 0.23 & $<0.001$ \\
\hline Digit span & $6.1 \pm 1.2(4-9)$ & $4.4 \pm 0.9(3-6)$ & $4.5 \pm 1.0(3-6)$ & $<0.001$ & $<0.001$ & 1.00 \\
\hline Spatial span & $5.6 \pm 1.0(4-7)$ & $2.5 \pm 1.2(0-4)$ & $3.4 \pm 1.6(0-5)$ & $<0.001$ & $<0.001$ & 0.08 \\
\hline Rey figure recall & $17.8 \pm 6.4(9.5-33)$ & $3.3 \pm 5.4(0-25.5)$ & $8.3 \pm 5.5(0-26)$ & $<0.001$ & $<0.001$ & 0.004 \\
\hline \multicolumn{7}{|l|}{ Visuospatial abilities } \\
\hline Rey figure copy & $32.6 \pm 2.6(27-36)$ & $12.2 \pm 10.1(5-32)$ & $20.0 \pm 9.7(1.5-36)$ & $<0.001$ & $<0.001$ & 0.005 \\
\hline Clock drawing test & - & $1.9 \pm 2.8(0-9)$ & $4.6 \pm 3.8(0-10)$ & - & - & 0.01 \\
\hline \multicolumn{7}{|l|}{ Attention and executive functions } \\
\hline Attentive matrices & $49.2 \pm 8.3(32-60)$ & $28.6 \pm 11.3(6-49)$ & $37.8 \pm 11.3(10-54)$ & $<0.001$ & 0.003 & 0.003 \\
\hline Raven's Coloured Progressive Matrices & $31.5 \pm 3.5(22-35)$ & $13.6 \pm 7.4(2-28)$ & $20.3 \pm 7.8(0-32)$ & $<0.001$ & $<0.001$ & 0.001 \\
\hline Semantic fluency & $43.2 \pm 7.8(27-56)$ & $17.9 \pm 8.8(5-40)$ & $22.2 \pm 11.1(0-48)$ & $<0.001$ & $<0.001$ & 0.20 \\
\hline Phonemic fluency & $38.2 \pm 10.8(18-55)$ & $15.1 \pm 9.3(2-39)$ & $13.6 \pm 11.0(0-37)$ & $<0.001$ & $<0.001$ & 1.00 \\
\hline Digit span backward & $4.8 \pm 0.9(3-6)$ & $2.3 \pm 1.0(0-4)$ & $3.4 \pm 1.0(2-5)$ & $<0.001$ & 0.001 & 0.01 \\
\hline \multicolumn{7}{|l|}{ Language } \\
\hline Token test & $34.3 \pm 1.6(30.5-36)$ & $24.6 \pm 6.2(10.5-34)$ & $27.1 \pm 5.6(13-36)$ & $<0.001$ & $<0.001$ & 0.22 \\
\hline
\end{tabular}

Abbreviations: CDR-SB = Clinical Dementia Rating Scale-Sum of Boxes; MMSE = Mini Mental State Examination; NPI = neuropsychiatry inventory . Values are number or mean $\pm S D$ (range). Disease duration is defined as years from onset to date of scan. $p$ Values refer to analysis of variance models, followed by post hoc pairwise comparisons (Bonferroni-corrected for multiple comparisons). 
METHODS Participants. Thirty-eight patients with a diagnosis of bvFTD, ${ }^{1} 37$ patients with a diagnosis of probable $\mathrm{AD}^{13}$ and age at onset $<65$ years (EOAD), and 32 age-matched healthy controls were recruited consecutively at the Scientific Institute and University Vita-Salute San Raffaele, Milan (table 1). The 2 patient groups were matched for disease duration and severity (table 1). Fourteen patients with bvFTD were included in a previous analysis. ${ }^{11}$ According to established criteria, ${ }^{1,13}$ the patient diagnoses were based on a comprehensive evaluation including clinical history, neurologic examination, neuropsychological testing, structural routine MRI, and CSF biomarkers. An experienced neurologist blinded to MRI results performed clinical assessments. Eligibility criteria included no family history of dementia; no (other) significant medical illnesses or substance abuse that could interfere with cognitive functioning; any other major systemic, psychiatric, or neurologic illnesses; and absence of other causes of focal or diffuse brain damage, including lacunae and extensive cerebrovascular disease at routine MRI. Healthy controls with no history of neurologic, psychiatric, or other major medical illnesses were recruited among friends and spouses of patients and by word of mouth.

Standard protocol approvals, registrations, and patient consents. The local ethical standards committee on human experimentation approved the study protocol and all participants (or their caregivers) provided written informed consent prior to study inclusion.

Neuropsychological assessment. Neuropsychological assessment was performed by an experienced neuropsychologist blinded to MRI results, and evaluated ${ }^{6}$ global cognitive functioning with the Mini-Mental State Examination (MMSE); long- and short-term verbal memory with the Rey immediate and delayed recall of 15 words and the digit span; long- and short-term spatial memory with the Rey figure delayed recall test and the spatial span; attentive and executive functions with the attentive matrices, the Raven colored progressive matrices, the digit span backward, and the phonemic and semantic fluency tests; visuospatial abilities with the Rey Figure Copy Test and the Clock drawing test; and language with the token test. In patients, the presence of behavioral and psychological symptoms of dementia was assessed using the neuropsychiatric inventory.

MRI analysis. Using a 3.0T Philips (Best, the Netherlands) Intera scanner, T2*-weighted single-shot echoplanar images for resting-state (RS) fMRI (repetition time/echo time $3,000 / 35 \mathrm{~ms}$, flip angle $90^{\circ}$, field of view $240 \mathrm{~mm}^{2}$, matrix $128 \times 128,200$ sets of 30, 4-mmthick axial slices) were acquired from all study participants. Appendix e-1 at Neurology.org reports the complete MRI protocol and details on RS AMRI preprocessing and construction of brain networks.

Global brain and lobar network analysis. Global and mean lobar network characteristics were explored using the Brain
Connectivity Matlab toolbox (brain-connectivity-toolbox.net). Network metrics, including clustering coefficient, characteristic path length, mean network strength, and local efficiency, were assessed to characterize the global topologic organization of global brain and lobar networks. ${ }^{14,15}$ In order to investigate the network characteristics in different areas of the brain, the 220 regions of interest from both hemispheres were grouped into 6 anatomic macro-areas (hereafter referred to as lobes): frontoinsular lobe, occipital lobe, temporal lobe, parietal lobe, basal ganglia, and sensorimotor area. Global and local metrics were compared between groups using analysis of variance models, followed by post hoc pairwise comparisons, Bonferroni-corrected for multiple comparisons $(p<0.05)$. In addition, in order to assess both interhemispheric and intrahemispheric lobar connectivity, we define the lobar connectivity weight (LCW) for each pair of lobes of the 2 hemispheres $\left(L_{x}, L_{y}\right)$ :

$$
\operatorname{LCW}\left(\mathrm{L}_{\mathrm{x}}, \mathrm{L}_{\mathrm{y}}\right)=\sum_{i \varepsilon L x, j \varepsilon L y} w_{i j},
$$

where $w_{i j}$ is the connectivity weight between regions $i$ and $j{ }^{16}$ $\mathrm{LCW}$ values were compared between groups by means of $t$ test, setting the level of significance at $p<0.05$ using network-based statistics (NBS). ${ }^{17,18}$ A corrected $p$ value was calculated for each pair of lobes using a permutation analysis (10,000 permutations). The direct comparisons between patients with EOAD and patients with bvFTD were adjusted for MMSE.

Regional connectivity analysis. NBS ${ }^{17,18}$ were performed to compare regional functional connectivity network data (in terms of Pearson correlation coefficients) between groups at the level of significance $p<0.05$. The largest (or principal) connected component and the smaller clusters of altered connections, which were not included in the principal connected component, ${ }^{17,18}$ were studied and displayed in the corresponding figures. A corrected $p$ value was calculated for each component using a permutation analysis (10,000 permutations). The direct comparison between patients with EOAD and patients with bvFTD was adjusted for MMSE.

Correlation analysis. Exploratory correlation analysis tested the relationship of global and lobar network metrics with dementia severity (Clinical Dementia Rating Scale-Sum of Boxes [CDR-SB]) and neuropsychological test scores of patients with EOAD and patients with bvFTD using the Pearson correlation ( $p<0.05$, uncorrected for multiple comparisons). The same clinical and cognitive scores were also correlated with functional connectivity data using NBS by means of Pearson correlations.

RESULTS Patients with EOAD relative to controls. Relative to controls, patients with EOAD showed severe global functional network alterations (lower mean nodal strength, local efficiency, and clustering

Table 2 Global graph analysis properties of brain network in in healthy controls (HC), patients with behavioral variant of frontotemporal dementia (bvFTD), and patients with early-onset Alzheimer disease (EOAD)

\begin{tabular}{|c|c|c|c|c|c|c|}
\hline & $\mathrm{HC}$ & EOAD & bvFTD & $\begin{array}{l}\text { p: EOAD } \\
\text { vs HC }\end{array}$ & $\begin{array}{l}\text { p: bvFTD } \\
\text { vs HC }\end{array}$ & $\begin{array}{l}\text { p: EOAD } \\
\text { vs bvFTD }\end{array}$ \\
\hline Nodal strength & $4.64 \pm 0.52(3.78-6.32)$ & $4.29 \pm 0.37$ (3.64-4.98) & $4.47 \pm 0.44(3.71-5.51)$ & 0.004 & 0.34 & 0.03 \\
\hline Local efficiency & $0.42 \pm 0.04(0.33-0.55)$ & $0.39 \pm 0.04(0.32-0.48)$ & $0.40 \pm 0.03(0.34-0.48)$ & 0.005 & 0.38 & 0.03 \\
\hline Clustering coefficient & $0.36 \pm 0.03(0.30-0.44)$ & $0.34 \pm 0.03(0.29-0.42)$ & $0.35 \pm 0.02(0.31-0.41)$ & 0.01 & 0.44 & 0.04 \\
\hline
\end{tabular}

Values are number or mean \pm SD (range). $p$ Values refer to analysis of variance models, followed by post hoc pairwise comparisons (Bonferroni-corrected for multiple comparisons). The direct comparison between patients with EOAD and patients with bvFTD was adjusted for Mini-Mental State Examination scores. 
coefficient, and longer mean path length) (table 2). The analysis of lobar network properties in patients with EOAD compared with controls showed a prominent involvement of the parietal, occipital, and frontoinsular lobes (alterations of all graph analysis metrics) (figure 1 and tables e- 1 and e-2). Furthermore, relative to controls, patients with EOAD showed a longer mean path length in the temporal lobe and reduced local efficiency and clustering coefficient in the sensorimotor regions. Patients with EOAD showed relative to controls a widespread pattern of reduced intrahemispheric LCW, involving temporal, frontoinsular, parietal, occipital, and sensorimotor lobes; and reduced interhemispheric LCW between right and left temporal lobes, right parietal and left temporal regions, and left parietal and right occipital lobes ( $p=0.01$; figure 2 and table e-3). At the regional connectivity level (NBS), compared to controls, patients with EOAD showed widespread functional connectivity reductions in pathways linking predominantly medial and lateral temporal, medial and lateral parietal, and medial frontoinsular nodes $(p<0.001$; figure 3$)$.

Patients with bvFTD relative to controls. Patients with bvFTD showed no global network abnormalities relative to controls (table 2). In the frontoinsular lobe, they showed a reduced nodal strength compared with controls (figure 1 and table e-2). Compared with controls, patients with bvFTD were characterized by a reduced intrahemispheric between-lobe LCW linking temporal, frontoinsular, parietal, and sensorimotor lobes bilaterally; reduced interhemispheric LCW between left and right temporal lobes; and increased interhemispheric LCW of left and right parietal lobes with basal ganglia and right sensorimotor and basal ganglia areas $(p=0.02$; figure 2 and table e-3). NBS analysis showed that patients with bvFTD relative to controls were characterized by a focal pattern of functional connectivity alterations including frontotemporal pathways and connections to the motor cortex and basal ganglia ( $p=0.03$; figure 3$)$.

Figure 1 Graph analysis properties of brain lobar networks in healthy controls $(\mathrm{HC})$, patients with behavioral variant of frontotemporal dementia (bvFTD), and patients with early-onset Alzheimer disease (EOAD)
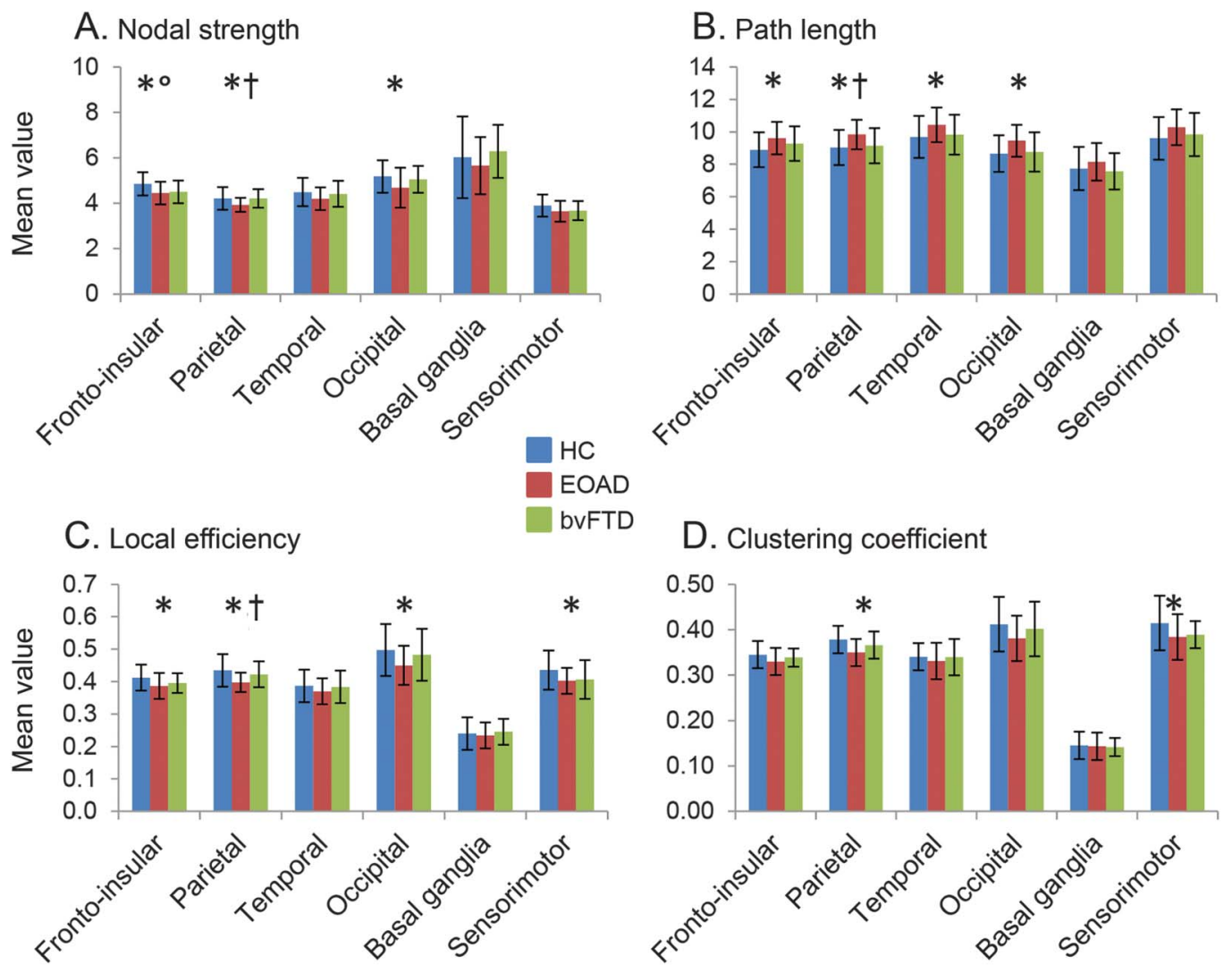

Mean values of nodal strength (A), path length (B), local efficiency $(C)$, and clustering coefficient (D) of each brain lobe for HC, patients with EOAD, and patients with bvFTD. Error bars are shown. $* p<0.05$ in patients with EOAD vs $H C ;{ }^{\circ} p<0.05$ in patients with bvFTD vs $\mathrm{HC}^{+}{ }^{+} p<0.05$ in patients with EOAD vs patients with bvFTD (see table e-3 for further details). The direct comparison between patients with EOAD and patients with bvFTD was adjusted for Mini-Mental State Examination scores. 
Figure 2 Intralobar and interlobar connectivity weights (LCW) in healthy controls (HC), patients with behavioral variant of frontotemporal dementia (bvFTD), and patients with early-onset Alzheimer disease (EOAD)

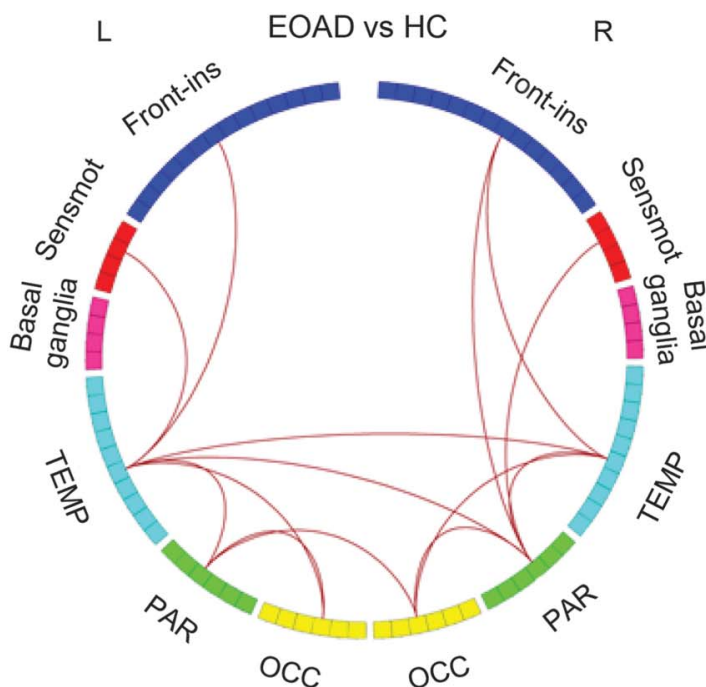

bvFTD vs HC
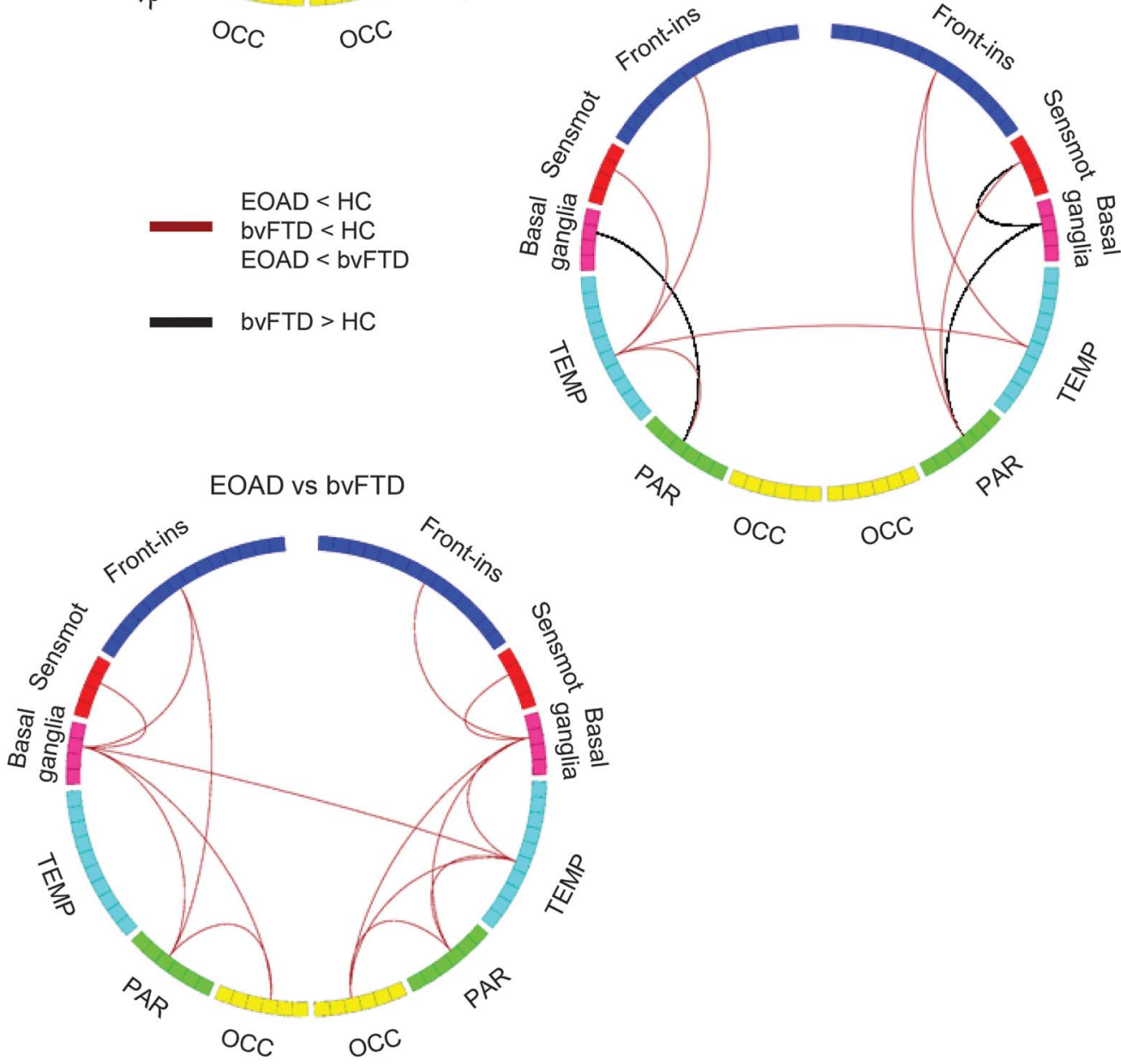

The figure shows the lobar regions arranged as a ring (the size of the regions being proportional to the number of brain nodes included; see table e-1 for further details). Red lines indicate decreased LCW in patients with EOAD relative to HC, patients with bvFTD relative to $\mathrm{HC}$, and patients with EOAD relative to patients with bvFTD. Black lines indicate increased LCW in patients with bvFTD compared with HC. The direct comparison between patients with EOAD and patients with bvFTD was adjusted for Mini-Mental State Examination scores. Front-ins = frontoinsular; OCC = occipital; PAR = parietal; Sensmot = sensorimotor; TEMP = temporal.

Patients with EOAD relative to patients with bvFTD. $\mathrm{Pa}$ tients with EOAD showed more severe global functional network alterations relative to patients with
bvFTD (table 2). In addition, patients with EOAD showed lower mean nodal strength, lower local efficiency, and longer mean path length of the parietal 
Figure 3 Affected functional connections in patients with early-onset Alzheimer disease (EOAD) and patients with behavioral variant of frontotemporal dementia (bvFTD) relative to healthy controls (HC) and each other (network-based statistic)

A. EOAD vs HC

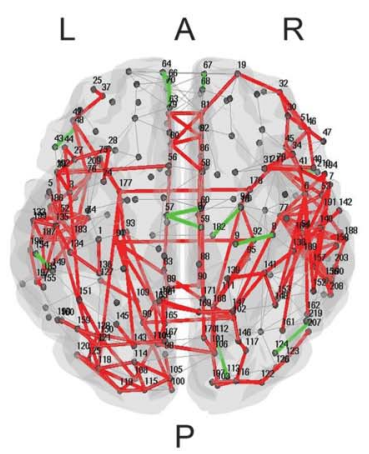

B. bvFTD vs HC

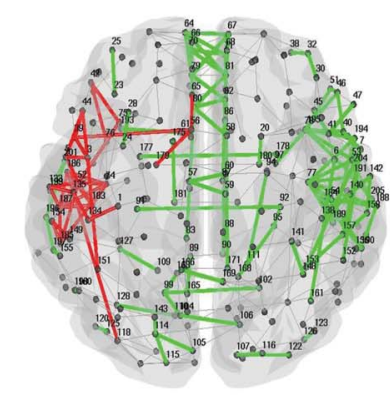

C. EOAD vs bvFTD

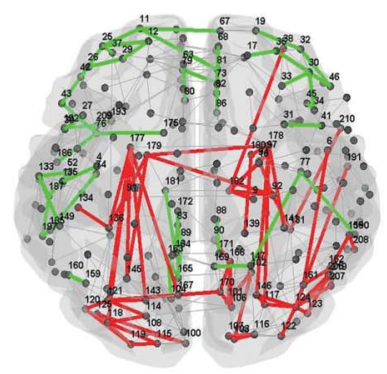

Subnetworks show reduced functional connectivity in (A) patients with EOAD relative to $H C$, (B) patients with bvFTD relative to $\mathrm{HC}$, and (C) patients with EOAD relative to patients with bvFTD. The direct comparison between patients with EOAD and patients with bvFTD was adjusted for Mini-Mental State Examination scores. The principal connected component is represented in red; the other affected connections not included in the principal connected component are shown in green. Table e-1 reports the names of each brain node with the corresponding number. $\mathrm{A}=$ anterior; $\mathrm{P}=$ posterior.

lobe relative to bvFTD cases (figure 1 and table e-2). The greater abnormalities of the parietal lobe in EOAD relative to bvFTD were observed also when only patients with disease duration $<3$ years were considered (MMSE-corrected $p$ values ranging from 0.001 to 0.01 ). Relative to patients with bvFTD, patients with $\mathrm{EOAD}$ were characterized by a reduced intrahemispheric LCW between temporal, parietal, occipital, frontoinsular, sensorimotor, and basal ganglia areas bilaterally, and a reduced interhemispheric LCW between right temporal lobe and left basal ganglia (MMSE-corrected $p=0.003$; figure 2 and table e-3). NBS showed that patients with EOAD relative to patients with bvFTD were characterized by a greater impairment of the functional connectivity in a network linking the hippocampus, medial and lateral parietal, medial and dorsolateral frontal, occipital and basal ganglia regions (MMSE-corrected $p<$ 0.001 ; figure 3 ). There were no connections with reduced functional connectivity in patients with bvFTD relative to patients with EOAD.

Clinical correlations. In patients with EOAD, CDR$\mathrm{SB}$ and neuropsychological test scores did not correlate with network measures. In patients with bvFTD, global network $(r=-0.35, p=0.049$ uncorrected) and frontal lobe $(r=-0.39, p=0.03$ uncorrected $)$ path length correlated with semantic fluency scores, while no association was found with CDR-SB scores. NBS analysis did not show significant correlations with clinical or cognitive data in either patient group.

DISCUSSION Using graph analysis and a connectomic approach to explore brain network functional connectivity in EOAD and bvFTD, we found that different clinical syndromes result in specific changes to brain networks. Global topologic organization of the functional brain network was significantly disrupted in patients with EOAD, while patients with bvFTD showed a relatively preserved global functional architecture. Patients with bvFTD were characterized by a focal involvement of frontoinsular and temporal regions. Functional connectivity breakdown in the posterior brain regions, particularly in the parietal lobe, differentiated patients with EOAD from patients with bvFTD. Finally, while EOAD was associated with widespread loss of both intrahemispheric and interhemispheric functional correlations, patients with bvFTD showed a preferential disruption of the intrahemispheric connectivity.

As compared with controls, patients with bvFTD showed reduced functional connectivity in the frontoinsular, temporal, and basal ganglia networks bilaterally, regions critical for social and emotional processing, task control, semantically driven personal evaluation, and maintenance of social decorum. This finding aligns with the selective, early vulnerability of frontoinsular, prefrontal, and temporal regions to frontotemporal lobar degeneration (FTLD) pathology $^{19}$ and previous evidence of functional alterations in the SN, executive control network, dorsolateral prefrontal attention network, and semantic appraisal network in bvFTD. ${ }^{6-8,20}$ Using graph analysis, frontoinsular hubs ${ }^{11}$ and decreased network centrality in the bilateral frontoinsular-temporal network ${ }^{12}$ have been reported in bvFTD. In a previous analysis, ${ }^{11}$ we showed that global topologic organization of the functional brain network in bvFTD was significantly disrupted relative to healthy controls. Although methodologic issues related to fMRI analysis might 
be a factor in determining these differences (see below), it is worth noting that the subgroup of patients with bvFTD included in the earlier analysis ${ }^{11}$ was clinically more impaired relative to the larger sample reported in the present study. This finding would suggest that the functional organization of brain networks in bvFTD worsens with advancing clinical severity, in keeping with the network-based degeneration hypothesis of neurodegenerative dementia. $^{21}$ Correlations observed in the present and previous studies ${ }^{11,12}$ between functional network alterations and executive dysfunction in bvFTD corroborate this network paradigm. Notably, we observed an association between functional network abnormalities and semantic fluency deficit in patients with bvFTD. Impaired fluency performance of patients with bvFTD has been previously related to an executive deficit in initiation which reduces rate of word production, leaving unaffected the semantic structure of the sequences produced. ${ }^{22}$ Taken together, these findings highlight how functional connectivity measures may serve as noninvasive markers of clinical decline in patients with bvFTD and underline the strategic importance of applying network analysis to understand the role of brain connectivity in disease evolution.

EOAD is characterized by a widespread loss of functional connection integrity throughout the brain, although parietal, occipital, and frontoinsular connections were preferentially affected. The extensive involvement of multiple functional connections observed in patients with EOAD might explain their early global cognitive impairment compared with late-onset $\mathrm{AD}$, including greater attention, executive, language, and visuospatial deficits. ${ }^{23}$ In addition, in EOAD intensified emotions can take the form of anxiety, irritability, and affective symptoms. ${ }^{23}$ Several imaging and pathologic studies of EOAD have demonstrated greater atrophy and pathologic burden in parietal and frontal cortex with relative sparing of hippocampus compared to patients with late-onset AD. ${ }^{24}$ Interestingly, patients with EOAD showed altered network features in the sensorimotor areas, which may represent an early functional deficit in a region that is typically affected latest in the disease process. In addition, most disrupted connections spanned across cortical lobes, indicating specific vulnerability of long-range connections. Several previous graph analysis studies in classic, late-onset $\mathrm{AD}^{25,26}$ and amnestic mild cognitive impairment ${ }^{27}$ showed a global decrease of functional connectivity, although other studies did not. ${ }^{28}$ In late-onset $\mathrm{AD}$, functional disconnection was primarily seen in the DMN regions, such as the precuneus, posterior cingulate, inferior parietal lobule, and temporal areas, which are known to manifest early AD pathology. ${ }^{26,27,29}$ Previous studies ${ }^{4,5}$ using independent component analysis in EOAD indicated reduced connectivity in the DMN, executive control, dorsolateral prefrontal, and language networks. Our findings are consistent but even more widespread than those of the previous studies.

Importantly, functional network topology and connectivity were different in EOAD and bvFTD. Patients with EOAD showed a more distributed and severe pattern of altered functional connectivity in an interconnected neural system including mainly hippocampal, parietal, and occipital regions. Notably, parietal lobe functional breakdown differentiated EOAD from bvFTD also considering patients in the early phase of the disease (disease duration $<3$ years). In $\mathrm{AD}$, amyloid deposits, tissue hypometabolism, and cortical atrophy all converge in posterior association cortices. ${ }^{30}$ Previous studies demonstrated the severe involvement of these regions especially in $\mathrm{EOAD},{ }^{24,31}$ in keeping with the severe involvement of posterior cortical cognitive functions such as word retrieval, visuospatial functions, arithmetic, and praxis. $^{23}$ In diametric contrast to EOAD, drawing, navigation, and other parietal lobe functions are retained or intensified in bvFTD until late-stage disease. ${ }^{32}$ Despite the clinical interest for the differential diagnosis between EOAD and bvFTD, few RS fMRI studies have compared the 2 dementias. ${ }^{6-8}$ In independent component analysis reports, patients with bvFTD have consistently shown decreased functional connectivity of the $\mathrm{SN}$ relative to $\mathrm{AD},{ }^{6,7}$ while findings in the posterior brain nodes have been inconsistent. ${ }^{6-8}$ Here we demonstrated the high sensitivity of graph-based analysis to detect disease-related disconnection patterns and its potential use to facilitate (and possibly improve) clinical diagnosis and fuel new insights into the syndromes' clinical diversity. fMRI data should be combined with structural MRI in future studies to test their relative contributions to patient classification.

Although we showed that between-lobe connectivity is affected in both dementias, we observed that patients with EOAD were characterized by loss of both intrahemispheric and interhemispheric functional correlations, while a preferential disruption of the intrahemispheric connectivity was found in bvFTD cases. Following the network paradigm, a specific disease phenotype emerges from the interaction between network architecture characteristics and the properties of abnormal protein that aggregate in that particular patient. ${ }^{33}$ With this in mind, we may speculate that AD- and FTLD-related proteins have a different effect on short- vs long-range connections or modulate differently the gradient of regional protein propagation across the vulnerable networks. ${ }^{33}$ In absence of pathologic data, longitudinal studies are required to confirm this possibility. 
Notably, in bvFTD but not in $\mathrm{AD}$, we observed not only deterioration of specific network connections, but also enhanced connectivity among the basal ganglia and relatively unaffected regions, such as parietal lobes and sensorimotor cortices. ${ }^{19,34}$ Previous fMRI studies of bvFTD showed the coexistence of both functional hypoconnectivity and hyperconnectivity. ${ }^{6,711,35,36}$ The classical explanation of this finding is that decreased functional connectivity is a direct effect of neurodegeneration, while increased connectivity may reflect neural compensation. However, this can be an oversimplification of a more complex scenario, in which increased connectivity could be also playing an active role in the pathogenesis of the disease. First, persistent hyperactivity/connectivity in some regions may place neurons under undue metabolic stress, reducing their viability and rendering them susceptible to degeneration..$^{37}$ In addition, it is plausible that increased connectivity in remote sites might also be caused by loss of cortical inhibitory influence. ${ }^{37}$

The study is not without limitations. Technically, network science applied to the human brain has yet to reach consensus regarding the best way to divide the brain into its most relevant anatomical units. ${ }^{38} \mathrm{We}$ defined as nodes 220 similarly sized regions covering the whole brain, excluding cerebellum. The merits of this strategy have been debated, ${ }^{39}$ but it is generally acknowledged that similarly sized regions of interest avoid larger regions to have higher connectivity because of their larger surface. Because parcellation strategies can markedly affect graph theoretical metrics, ${ }^{38,40}$ comparisons with previous studies using different approaches ${ }^{11}$ can be challenging. Second, although RS fMRI data were carefully registered to and masked with gray matter maps to avoid a regional atrophy influence, a possible partial volume effect on our results cannot be excluded. Third, the diagnosis of neurodegenerative dementia can only be confirmed by autopsy. Postmortem evaluation was not available in our study; therefore, the possibility of misdiagnosis cannot be excluded. Nevertheless, all patients underwent an extensive dementia screening and were evaluated in a multidisciplinary panel including clinicians specialized in dementia. Fourth, correlation analysis was not corrected for multiple comparisons. Although an association between executive dysfunction and functional network abnormalities in bvFTD has been reported, ${ }^{11,12}$ we cannot exclude false-positive findings. Finally, this is a cross-sectional study. Additional longitudinal studies are needed to assess whether the functional brain organization changes differently with the progression of the 2 diseases.

Our study shows that the approach of characterizing the brain as a network using RS fMRI and graph theoretical analysis can provide new insights into how neurodegeneration affects brain function in different early-onset neurodegenerative dementias. Future studies that integrate different imaging modalities will be helpful to clarify whether the disease-specific patterns of functional changes in EOAD and bvFTD are associated with specific structural connectivity alterations.

\section{AUTHOR CONTRIBUTIONS}

M. Filippi and F.A. were responsible for study conception and design. M. Filippi, S.B., E.C., F.I., A.M., F.C., G.M., M. Falautano, and F.A. were responsible for acquisition and analyses of data. All authors were responsible for drafting or revising the text.

\section{ACKNOWLEDGMENT}

The authors thank the patients and their families for participation.

\section{STUDY FUNDING}

The study was supported by the Italian Ministry of Health (grant GR2010-2303035) and Alzheimer's Drug Discovery Foundation (grant 20131211).

\section{DISCLOSURE}

M. Filippi is Editor-in-Chief of the Journal of Neurology; serves on a scientific advisory board for Teva Pharmaceutical Industries; has received compensation for consulting services and/or speaking activities from Biogen Idec, ExceMED, Novartis, and Teva Pharmaceutical Industries; and receives research support from Biogen Idec, Teva Pharmaceutical Industries, Novartis, Italian Ministry of Health, Fondazione Italiana Sclerosi Multipla, Cure PSP, Alzheimer's Drug Discovery Foundation (ADDF), the Jacques and Gloria Gossweiler Foundation (Switzerland), and ARiSLA (Fondazione Italiana di Ricerca per la SLA). S. Basaia reports no disclosures relevant to the manuscript. E. Canu has received research support from the Italian Ministry of Health. F. Imperiale, A. Meani, F. Caso, G. Magnani, and M. Falautano report no disclosures relevant to the manuscript. G. Comi has received consulting fees for participating on advisory boards from Novartis, Teva Pharmaceutical Industries, Sanofi, Genzyme, Merck Serono, Bayer, and Actelion, and honorarium for speaking activities from Novartis, Teva Pharmaceutical Industries, Sanofi, Genzyme, Merck Serono, Bayer, Biogen, and ExceMED. A. Falini reports no disclosures relevant to the manuscript. F. Agosta is Section Editor of NeuroImage: Clinical; has received speaker honoraria from ExceMED-Excellence in Medical Education and Biogen Idec; and receives or has received research support from the Italian Ministry of Health, AriSLA (Fondazione Italiana di Ricerca per la SLA), and the European Research Council. Go to Neurology.org for full disclosures.

Received April 10, 2017. Accepted in final form July 19, 2017.

\section{REFERENCES}

1. Rascovsky K, Hodges JR, Knopman D, et al. Sensitivity of revised diagnostic criteria for the behavioural variant of frontotemporal dementia. Brain 2011;134:2456-2477.

2. Agosta F, Pievani M, Geroldi C, Copetti M, Frisoni GB, Filippi M. Resting state fMRI in Alzheimer's disease: beyond the default mode network. Neurobiol Aging 2012;33:1564-1578.

3. Jones DT, Knopman DS, Gunter JL, et al. Cascading network failure across the Alzheimer's disease spectrum. Brain 2016;139:547-562.

4. Gour N, Felician O, Didic M, et al. Functional connectivity changes differ in early and late-onset Alzheimer's disease. Hum Brain Mapp 2014;35:2978-2994.

5. Lehmann M, Madison C, Ghosh PM, et al. Loss of functional connectivity is greater outside the default mode network in nonfamilial early-onset Alzheimer's disease variants. Neurobiol Aging 2015;36:2678-2686. 
6. Filippi M, Agosta F, Scola E, et al. Functional network connectivity in the behavioral variant of frontotemporal dementia. Cortex 2013;49:2389-2401.

7. Zhou J, Greicius MD, Gennatas ED, et al. Divergent network connectivity changes in behavioural variant frontotemporal dementia and Alzheimer's disease. Brain 2010; 133:1352-1367.

8. Hafkemeijer A, Moller C, Dopper EG, et al. Resting state functional connectivity differences between behavioral variant frontotemporal dementia and Alzheimer's disease. Front Hum Neurosci 2015;9:474.

9. Bullmore E, Sporns O. Complex brain networks: graph theoretical analysis of structural and functional systems. Nat Rev Neurosci 2009;10:186-198.

10. Tijms BM, Wink AM, de Haan W, et al. Alzheimer's disease: connecting findings from graph theoretical studies of brain networks. Neurobiol Aging 2013;34:2023-2036.

11. Agosta F, Sala S, Valsasina P, et al. Brain network connectivity assessed using graph theory in frontotemporal dementia. Neurology 2013;81:134-143.

12. Sedeno L, Couto B, Garcia-Cordero I, et al. Brain network organization and social executive performance in frontotemporal dementia. J Int Neuropsychol Soc 2016;22:250-262.

13. McKhann GM, Knopman DS, Chertkow H, et al. The diagnosis of dementia due to Alzheimer's disease: recommendations from the National Institute on Aging-Alzheimer's Association workgroups on diagnostic guidelines for Alzheimer's disease. Alzheimers Dement 2011;7:263-269.

14. Watts DJ, Strogatz SH. Collective dynamics of "smallworld" networks. Nature 1998;393:440-442.

15. Sporns O, Chialvo DR, Kaiser M, Hilgetag CC. Organization, development and function of complex brain networks. Trends Cogn Sci 2004;8:418-425.

16. Ingalhalikar M, Smith A, Parker D, et al. Sex differences in the structural connectome of the human brain. Proc Natl Acad Sci USA 2014;111:823-828.

17. Zalesky A, Fornito A, Bullmore ET. Network-based statistic: identifying differences in brain networks. Neuroimage 2010;53:1197-1207.

18. Galantucci S, Agosta F, Stefanova E, et al. Structural brain connectome and cognitive impairment in Parkinson disease. Radiology 2017;283:515-525.

19. Seeley WW, Crawford R, Rascovsky K, et al. Frontal paralimbic network atrophy in very mild behavioral variant frontotemporal dementia. Arch Neurol 2008;65:249-255.

20. Ranasinghe KG, Rankin KP, Pressman PS, et al. Distinct subtypes of behavioral variant frontotemporal dementia based on patterns of network degeneration. JAMA Neurol 2016;73:1078-1088.

21. Seeley WW, Crawford RK, Zhou J, Miller BL, Greicius MD. Neurodegenerative diseases target large-scale human brain networks. Neuron 2009;62:42-52.

22. Reverberi C, Cherubini P, Baldinelli S, Luzzi S. Semantic fluency: cognitive basis and diagnostic performance in focal dementias and Alzheimer's disease. Cortex 2014;54:150-164.

23. Smits LL, Pijnenburg YA, Koedam EL, et al. Early onset Alzheimer's disease is associated with a distinct neuropsychological profile. J Alzheimers Dis 2012;30:101-108.
24. Canu E, Agosta F, Spinelli EG, et al. White matter microstructural damage in Alzheimer's disease at different ages of onset. Neurobiol Aging 2013;34:2331-2340.

25. Supekar K, Menon V, Rubin D, Musen M, Greicius MD. Network analysis of intrinsic functional brain connectivity in Alzheimer's disease. PLoS Comput Biol 2008;4: e1000100.

26. Zhao X, Liu Y, Wang X, et al. Disrupted small-world brain networks in moderate Alzheimer's disease: a resting-state FMRI study. PLoS One 2012;7:e33540.

27. Minati L, Chan D, Mastropasqua C, et al. Widespread alterations in functional brain network architecture in amnestic mild cognitive impairment. J Alzheimers Dis 2014; 40:213-220.

28. Sanz-Arigita EJ, Schoonheim MM, Damoiseaux JS, et al. Loss of "small-world" networks in Alzheimer's disease: graph analysis of FMRI resting-state functional connectivity. PLoS One 2010;5:e13788.

29. Dai Z, Yan C, Li K, et al. Identifying and mapping connectivity patterns of brain network hubs in Alzheimer's disease. Cereb Cortex 2015;25:3723-3742.

30. Buckner RL, Snyder AZ, Shannon BJ, et al. Molecular, structural, and functional characterization of Alzheimer's disease: evidence for a relationship between default activity, amyloid, and memory. J Neurosci 2005;25:77097717.

31. Ossenkoppele R, Zwan MD, Tolboom N, et al. Amyloid burden and metabolic function in early-onset Alzheimer's disease: parietal lobe involvement. Brain 2012;135:21152125 .

32. Mendez MF, Cherrier M, Perryman KM, Pachana N, Miller BL, Cummings JL. Frontotemporal dementia versus Alzheimer's disease: differential cognitive features. Neurology 1996;47:1189-1194.

33. Warren JD, Rohrer JD, Schott JM, Fox NC, Hardy J, Rossor MN. Molecular nexopathies: a new paradigm of neurodegenerative disease. Trends Neurosci 2013;36: 561-569.

34. Brambati SM, Renda NC, Rankin KP, et al. A tensor based morphometry study of longitudinal gray matter contraction in FTD. Neuroimage 2007;35:998-1003.

35. Whitwell JL, Josephs KA, Avula R, et al. Altered functional connectivity in asymptomatic MAPT subjects: a comparison to bvFTD. Neurology 2011;77:866-874.

36. Farb NA, Grady CL, Strother S, et al. Abnormal network connectivity in frontotemporal dementia: evidence for prefrontal isolation. Cortex 2013;49:1856-1873.

37. Fornito A, Zalesky A, Breakspear M. The connectomics of brain disorders. Nat Rev Neurosci 2015;16:159-172.

38. Wig GS, Schlaggar BL, Petersen SE. Concepts and principles in the analysis of brain networks. Ann NY Acad Sci 2011;1224:126-146.

39. Zalesky A, Fornito A, Harding IH, et al. Whole-brain anatomical networks: does the choice of nodes matter? Neuroimage 2010;50:970-983.

40. Fornito A, Zalesky A, Bullmore ET. Network scaling effects in graph analytic studies of human resting-state FMRI data. Front Syst Neurosci 2010;4:22. 


\section{Neurology}

\section{Brain network connectivity differs in early-onset neurodegenerative dementia Massimo Filippi, Silvia Basaia, Elisa Canu, et al.}

Neurology 2017;89;1764-1772 Published Online before print September 27, 2017

DOI 10.1212/WNL.0000000000004577

This information is current as of September 27, 2017

\section{Updated Information \& Services \\ Supplementary Material}

\section{References}

Citations

Subspecialty Collections

Permissions \& Licensing

Reprints including high resolution figures, can be found at: http://n.neurology.org/content/89/17/1764.full

Supplementary material can be found at: http://n.neurology.org/content/suppl/2017/09/27/WNL.0000000000004 577.DC1

http://n.neurology.org/content/suppl/2017/09/27/WNL.0000000000004 577.DC2

This article cites 40 articles, 5 of which you can access for free at: http://n.neurology.org/content/89/17/1764.full\#ref-list-1

This article has been cited by 4 HighWire-hosted articles: http://n.neurology.org/content/89/17/1764.full\#\#otherarticles

This article, along with others on similar topics, appears in the following collection(s):

Alzheimer's disease

http://n.neurology.org/cgi/collection/alzheimers_disease

Frontotemporal dementia

http://n.neurology.org/cgi/collection/frontotemporal_dementia MRI

http://n.neurology.org/cgi/collection/mri

Information about reproducing this article in parts (figures,tables) or in its entirety can be found online at:

http://www.neurology.org/about/about_the_journal\#permissions

Information about ordering reprints can be found online:

http://n.neurology.org/subscribers/advertise

Neurology ${ }^{\circledR}$ is the official journal of the American Academy of Neurology. Published continuously since 1951, it is now a weekly with 48 issues per year. Copyright Copyright ( 2017 The Author(s). Published by Wolters Kluwer Health, Inc. on behalf of the American Academy of Neurology.. All rights reserved. Print ISSN: 0028-3878. Online ISSN: 1526-632X.

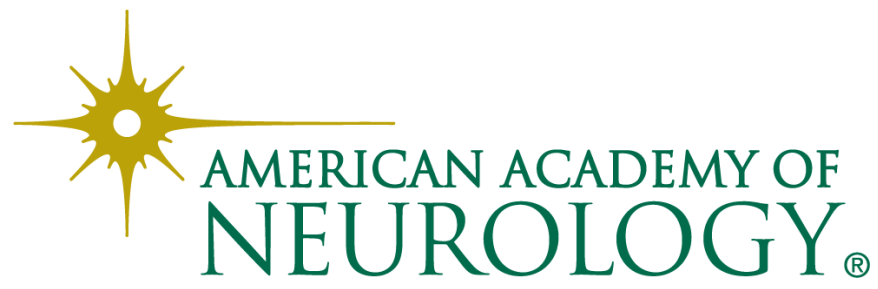

